\title{
Network Pharmacological Study of Yiyi-Fuzi-Baijiang powder in Treating Colorectal Cancer
}

\begin{abstract}
X. WANG, Z. G. SUN ${ }^{1}$, Y. LU², Y. Q. XU* AND L. Q. XIE ${ }^{3^{*}}$
Department of Oncology, the Second Affiliated Hospital of Nanjing University of Chinese Medicine, No. 23, Nanhu Road, Nanjing, Jiangsu 210017, China, ${ }^{1}$ Key Laboratory for Metabolic Diseases in Chinese Medicine, First Clinical Medical College, Nanjing University of Chinese Medicine, No. 155 Hanzhong Road, Nanjing Jiangsu 210023, China, ${ }^{2}$ Jiangsu Key Laboratory for Efficacy and Safety Evaluation of Chinese Material Medical, School of Pharmacy, Nanjing University of Chinese Medicine, No. 138 Xianlin Avenue, Nanjing Jiangsu 210023, China, ${ }^{3}$ Department of Traditional Chinese Medicine, The First Affiliated Hospital of Nanjing Medical University, No. 300, Guangzhou Road, Nanjing, Jiangsu 210029, China
\end{abstract}

Wang et al.: Pharmacological Study of Yiyi-Fuzi-Baijiang powder

\begin{abstract}
This investigation dealt with the analysis of the active ingredients of Yiyi-Fuzi-Baijiang powder in the treatment of colorectal cancer using computer network pharmacology technology to predict the mechanism of action. The chemical constituents and targets of Yiyi-Fuzi-Baijiang powder were obtained from Traditional Chinese Medicine Systems Pharmacology Database and Analysis Platform and the common targets in the treatment of colorectal cancer were obtained from Online Mendelian Inheritance in Man, Therapeutic Target Database and PharmGkb database. Excel was used to screen the molecular target and Cytoscape software was used to establish the network of Chinese medicine components target of Yiyi-Fuzi-Baijiang powder. The gene function and metabolic pathway were analyzed using the biological information annotation database. Nineteen components of Yiyi-Fuzi-Baijiang powder were found to interact with 121 target proteins of colorectal cancer, including progesterone receptor, prostaglandin G/H synthase 2, gamma-aminobutyric acid receptor subunit alpha-1, prostaglandin $G / H$ synthase 1 , nuclear receptor co-activator 2, beta-2 adrenergic receptor, sodium channel protein type 5 subunit alpha, alpha-1B adrenergic receptor. It is the target of Chaihushugansan in the treatment of post-stroke depression, mainly involving in neuroactive ligand-receptor interaction, endocrine regulated calcium reabsorption, TGF- $\beta$ signaling pathway, Hedgehog signaling pathway, inflammatory mediator regulation transient receptor potential channels, cholinergic synapse, through the regulation of the nervous system, cell cycle, apoptosis, inflammatory regulation, cell communication and other biological processes to treat colorectal cancer. The possible mechanism of Yiyi-Fuzi-Baijiang powder in treating colorectal cancer was revealed by network pharmacological study, which laid a foundation for further elucidation of its action target.
\end{abstract}

Key words: Coix seed, monkshood, Patrinia villosa, colorectal cancer, network pharmacology

Colorectal cancer, including colorectal and rectal cancer, is the third most common cause of cancer death in the world. Its 5 y survival rate is only 30 $65 \%{ }^{[1]}$. In recent years, the incidence of colorectal cancer has increased gradually in developing countries. The incidence and mortality of colorectal cancer in China ranks fifth and sixth, respectively among all the malignant tumours in the urban population, which has seriously threatened the health of people in China ${ }^{[2]}$. Its prevention and treatment has become a hot research topic $^{[3-5]}$. Colorectal cancer in traditional Chinese clinical medicine clinical was grouped under intestinal Qin, accumulation, locking anal hemorrhoids, visceral toxin and scattered in enteropathy, intestinal wind, diarrhoea, colon Yin, intestinal wind blood and other diseases. The treatment outcomes of traditional Chinese medicine is significant ${ }^{[6-8]}$. The early clinical research and animal experiment of the research group confirmed that Yiyi-Fuzi-Baijiang powder showed good curative effect in the treatment of colorectal cancer ${ }^{[9]}$. However, due

*Address for correspondence

E-mail: xie-liqun@163.com 
to the regulation of multi-component and multi-target of traditional Chinese medicine, the mechanism of Yiyi-Fuzi-Baijiang powder in the treatment of colorectal cancer is not clear.

Network pharmacology is a new method to study the mechanism of traditional Chinese medicinal compounds ${ }^{[10]}$. The purpose of this study is to explore the potential effective components and possible mechanism of Yiyi-Fuzi-Baijiang powder in the treatment of colorectal cancer and to systematically explore the overall regulatory effect of the multi-component and multi-target drug on colorectal cancer at the molecular level, so as to provide a basis for the further research and development of compounds in traditional Chinese medicine.

\section{MATERIALS AND METHODS}

Traditional Chinese medicine systems pharmacology (TCMSP) is a platform integrating pharmacokinetics, pharmaceutical chemistry and drug target protein network disease network ${ }^{[11]}$. Based on the TCMSP database, the chemical constituents of Coix, aconite and Patrinia villosa were searched. All the chemical components were screened by Excel and the component data were obtained.

\section{Research tools:}

TCMSP; UniProt Database to obtain gene information of components, targets, interrelations and targets of traditional Chinese Medicine; Online Frontal Analysis Mendelian Inheritance in Man (OMIM) ${ }^{[12,13]}$, Drug target database, Databases/TTD/TTD.asp and PharmGkb database acquisition of target proteins for stroke and depression; Cytoscape 3.2.1 software building a network of compound target interaction ${ }^{[12-16]}$.

\section{Screening of active compounds of Yiyi-Fuzi- Baijiang powder:}

The target organs and tissues for traditional Chinese medicine should be achieved through the process of absorption, distribution, metabolism and excretion (ADME). Oral bioavailability (OB), drug likeness (DL), blood-brain barrier (BBB) and half-life (HL) are the key parameters of ADME. In this study, TCMSP data platform was used to evaluate $\mathrm{OB}, \mathrm{DL}, \mathrm{BBB}$ and $\mathrm{HL}$ of each active component of Yiyi-Fuzi-Baijiang powder and selected chemical components that simultaneously meet the requirements of $\mathrm{OB} \geq 30 \%, \mathrm{DL} \geq 0.18$, BBB $\geq 0.3$ and $\mathrm{HL} \geq 4 \mathrm{~h}$ as the candidate active components.

\section{Target selection of Yiyi-Fuzi-Baijiang powder in the treatment of colorectal cancer:}

GADCC, therapeutic target database (TTD) and PharmGKB databases were used to search the coding genes of colorectal cancer to determine the disease target. Based on the molecular docking technology, the effective components and disease targets of YiyiFuzi-Baijiang powder were screened and the target of Yiyi-Fuzi-Baijiang powder in the treatment of colorectal cancer was obtained.

\section{Biological process and metabolic pathway analysis of target:}

Database for annotation, visualization and integrated discovery (DAVID) can provide annotated information of biological functions and find the most significant biological annotation. By inputting the list of target gene names and defining the species as human, and correcting all the target gene names to their official names, the enrichment analysis of go biological process and the metabolism of Kyoto Encyclopaedia of genes and genomes (KEGG) pathway analysis were carried out pathway enrichment analysis.

\section{RESULTS AND DISCUSSION}

The results showed that there were 19 active molecules in baizao powder that included 6 , sitosterol $\alpha 1$, mandenol, (2R)-2,3-dihydroxypropyl(z)-octadec9-enoate, sitosterol, stigmasterol and CLR; 6, 11,14-eicosadienoic acid, deltoin, karakoline, karanjin, neonadsuranic acid $\mathrm{B}$, sitosterol and 7 of Patrinia villosa $\mathrm{L}$, morrianine $\mathrm{C}$, asperglauccide, acacetin, sinoacutine, $\beta$-sitosterol, sitosterol, and stigmasterol (Table 1). Through UniProt database, the target points of the above ingredients are transformed into gene names, and the composition target map of traditional Chinese medicine is constructed (fig. 1).

Using GADCC, TTD and PharmGKB database to search the coding genes of colorectal cancer, there are 121 targets as disease targets (Table 2). Based on the molecular docking technology, 19 active ingredients and 121 disease targets of Coix Fuzi baizao powder were screened, and 8 well docking targets were obtained as the targets of Coix Fuzi baizao powder in the treatment of colorectal cancer (Table 3 ), respectively progesterone receptor, prostaglandin $\mathrm{G} / \mathrm{H}$ synthase 2 , $\gamma$-aminobutyric acid receptor subunit -1 , prostaglandin $\mathrm{G} / \mathrm{H}$ synthase 1 , nuclear receptor coactivator $2, \beta-2$ adrenergic receptor, sodium channel protein type 5 subunit $\alpha, \alpha-1 \mathrm{~B}$ adrenergic receptor. 
TABLE 1: CANDIDATE ACTIVE COMPONENTS AND PHARMACOKINETIC PARAMETERS OF YIYI-FUZIBAIJIANG POWDER

\begin{tabular}{lcccccc}
\hline Number & Component & OB/\% & DL & BBB & HL & Medicinal Materials \\
\hline MOL001323 & Sitosterol $\alpha 1$ & 43.28 & 0.78 & 0.97 & 5.64 & Coix seed \\
MOL001494 & Mandenol & 42 & 0.19 & 1.14 & 5.39 & Coix seed \\
MOL002882 & (2R)-2,3-dihydroxypropyl (Z)-octadec- & 34.13 & 0.3 & -0.22 & 5.19 & Coix seed \\
MOL000359 & 9-enoate & 36.91 & 0.75 & 0.87 & 5.37 & Coix seed \\
MOL000449 & Sitosterol & 43.83 & 0.76 & 1 & 5.57 & Coix seed \\
MOL000953 & Stigmasterol & 37.87 & 0.68 & 1.13 & 4.52 & Coix seed \\
MOL002211 & CLR & 39.99 & 0.2 & 0.76 & 5.6 & monkshood \\
MOL002392 & 11,14-eicosadienoic acid & 46.69 & 0.37 & -0.12 & 7.7 & monkshood \\
MOL002397 & Deltoin & 51.73 & 0.73 & -0.03 & 11.1 & monkshood \\
MOL002398 & Karakoline & 69.56 & 0.34 & 0.62 & 13.15 & monkshood \\
MOL002401 & Karanjin & 43.1 & 0.85 & -0.01 & 12.05 & monkshood \\
MOL000359 & Neokadsuranic acid B & 36.91 & 0.75 & 0.87 & 5.37 & monkshood \\
MOL001676 & Sitosterol & 33.96 & 0.22 & 0.14 & 21.71 & Patrinia villosa \\
MOL001677 & Vilmorrianine C & 58.02 & 0.52 & -0.22 & 6.88 & Patrinia villosa \\
MOL001689 & Asperglaucide & 34.97 & 0.24 & -0.05 & 17.25 & Patrinia villosa \\
MOL001697 & Acacetin & 63.39 & 0.53 & 0.36 & 4.9 & Patrinia villosa \\
MOL000358 & Sinoacutine & 36.91 & 0.75 & 0.99 & 5.36 & Patrinia villosa \\
MOL000359 & B-sitosterol & 36.91 & 0.75 & 0.87 & 5.37 & Patrinia villosa \\
MOL000449 & Sitosterol & 43.83 & 0.76 & 1 & 5.57 & Patrinia villosa \\
\hline
\end{tabular}

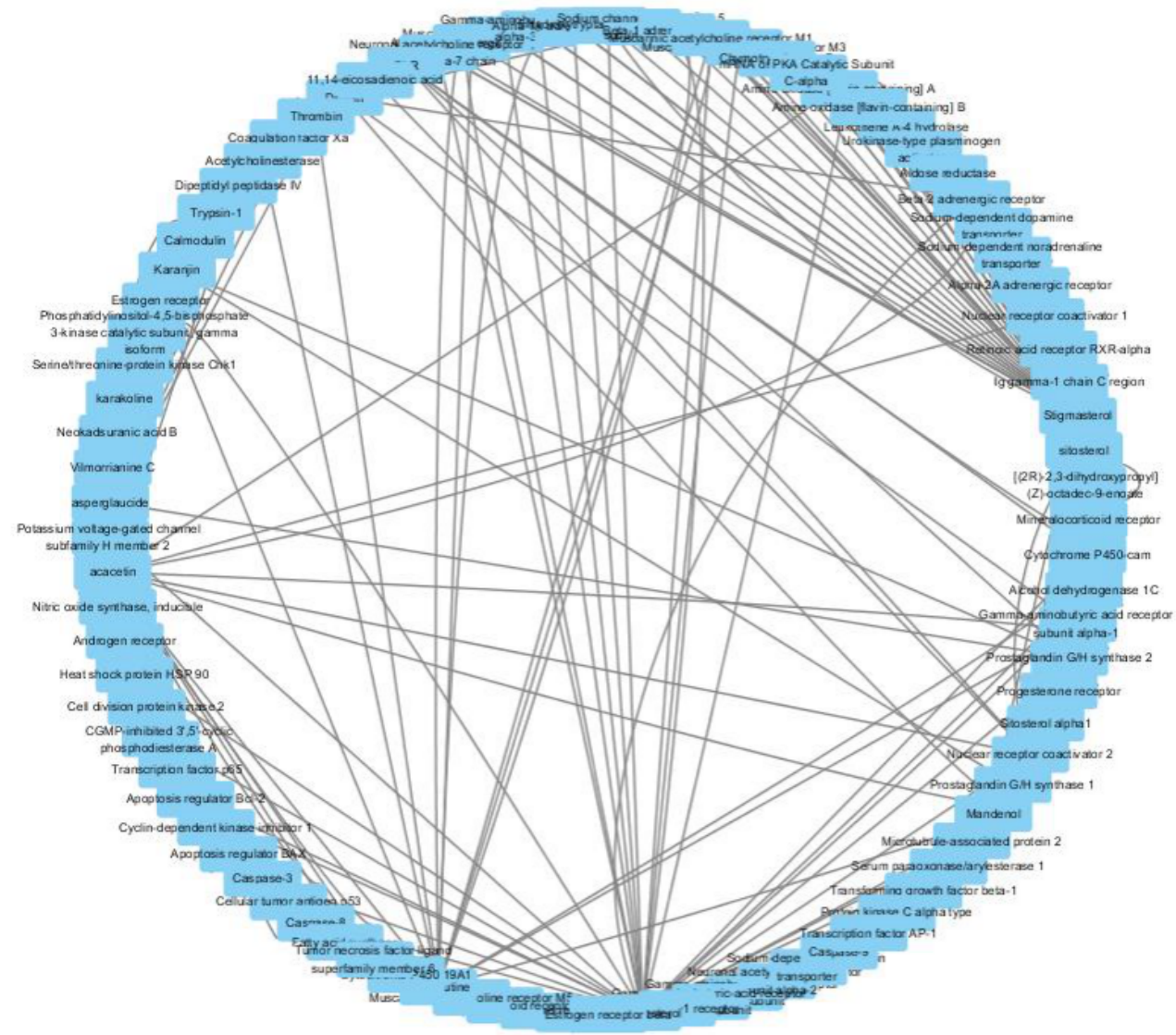

Fig. 1: Composition target map of Yiyi-Fuzi-Baijiang powder

The results showed that the 8 predicted targets were related to biological processes such as neural regulation, cell cycle, apoptosis, inflammatory regulation, cell communication and so on (Table 4). It is suggested that Yiyi-Fuzi-Baijiang powder may play an anticancer role by improving these biological processes. The results of 
www.ijpsonline.com

TABLE 2: DISEASE TARGETS

\begin{tabular}{|c|c|c|c|}
\hline 1 & $\begin{array}{c}\text { Vascular endothelial growth factor } \\
\text { receptor } 1\end{array}$ & 65 & PTGS1 \\
\hline 2 & $\begin{array}{c}\text { Vascular endothelial growth factor } \\
\text { receptor } 2\end{array}$ & 66 & PTGS2 \\
\hline 3 & $\begin{array}{c}\text { Potassium-transporting ATPase } \alpha \\
\text { chain } 1\end{array}$ & 67 & ADRB2 \\
\hline 4 & Retinoic acid receptor $\alpha$ & 68 & $\mathrm{ADH} 1 \mathrm{~B}$ \\
\hline 5 & DNA topoisomerase I & 69 & $\mathrm{FH}$ \\
\hline 6 & DNA topoisomerase II & 70 & ZFHX3 \\
\hline 7 & FL cytokine receptor & 71 & MXI1 \\
\hline 8 & Histone deacetylase 1 & 72 & ADRA1B \\
\hline 9 & Tumor necrosis factor & 73 & EIF4G1 \\
\hline 10 & Calcium channel & 74 & OPCML \\
\hline 11 & MLH3 & 75 & NKX2-1 \\
\hline 12 & AXIN2 & 76 & GABRA-1 \\
\hline 13 & MCC & 77 & PTPRJ \\
\hline 14 & PIK3CA & 78 & HMMR \\
\hline 15 & AKT1 & 79 & SMAD4 \\
\hline 16 & FGFR3 & 80 & ATR \\
\hline 17 & MSH6 & 81 & ACVR1B \\
\hline 18 & CHEK2 & 82 & BARD1 \\
\hline 19 & TP53 & 83 & NCOA-2 \\
\hline 20 & MUTYH & 84 & STK11 \\
\hline 21 & DCC & 85 & PRKN \\
\hline 22 & BRAF & 86 & FOXE1 \\
\hline 23 & PMS2 & 87 & MAD1L1 \\
\hline 24 & DLC1 & 88 & PHB \\
\hline 25 & PDGFRL & 89 & RAD51C \\
\hline 26 & CTNNB1 & 90 & FGFR2 \\
\hline 27 & MSH2 & 91 & RAD51D \\
\hline 28 & APC & 92 & AURKA \\
\hline 29 & MLH1 & 93 & PPP2R1B \\
\hline 30 & TGFBR2 & 94 & RAD54L \\
\hline 31 & NRAS & 95 & HABP2 \\
\hline 32 & CCND1 & 96 & RAD54B \\
\hline 33 & PLA2G2A & 97 & SCN5A \\
\hline 34 & BAX & 98 & ELAC2 \\
\hline 35 & POLE & 99 & RAD51 \\
\hline 36 & POLD1 & 100 & BRIP1 \\
\hline 37 & BUB1 & 101 & RNASEL \\
\hline 38 & EP300 & 102 & IL1RN \\
\hline 39 & BUB1B & 103 & IL1B \\
\hline 40 & SMAD7 & 104 & SRGAP1 \\
\hline 41 & TLR2 & 105 & RB1CC1 \\
\hline 42 & FLCN & 106 & MPO \\
\hline 43 & GALNT12 & 107 & ATM \\
\hline 44 & EPCAM & 108 & SASH1 \\
\hline 45 & BRCA2 & 109 & PALLD \\
\hline 46 & KRAS & 110 & ERCC6 \\
\hline 47 & $\mathrm{CDH} 1$ & 111 & EHBP1 \\
\hline 48 & BRCA1 & 112 & CHRNA3 \\
\hline
\end{tabular}

\begin{tabular}{lccc}
49 & EGFR & 113 & CHRNA5 \\
50 & ERBB2 & 114 & RNF43 \\
51 & CDKN2A & 115 & SDCCAG8 \\
52 & EPHB2 & 116 & CYP2A6 \\
53 & PTEN & 117 & RHBDF2 \\
54 & CASP8 & 118 & MSMB \\
55 & KLF6 & 119 & HRAS \\
56 & SLC22A18 & 120 & SRC \\
57 & SLC22A18 & 121 & NQ01 \\
58 & PPM1D & & \\
59 & IRF1 & & \\
60 & AR & & \\
61 & PALB2 & & \\
62 & RB1 & & \\
63 & MAP3K8 & & \\
64 & PGR & & \\
\hline
\end{tabular}

TABLE 3: POTENTIAL TARGET OF YIYI-FUZIBAIJIANG POWDER IN THE TREATMENT OF COLORECTAL CANCER

\begin{tabular}{|c|c|c|c|}
\hline number & \multicolumn{2}{|c|}{ Target protein } & Gene name \\
\hline MOL000359 & \multicolumn{2}{|c|}{ Progesterone receptor } & PGR \\
\hline MOL000449 & \multicolumn{2}{|c|}{ Prostaglandin $\mathrm{G} / \mathrm{H}$ synthase 2} & PTGS2 \\
\hline MOL000449 & \multicolumn{2}{|c|}{$\begin{array}{l}\text { Gamma-aminobutyric acid } \\
\text { receptor subunit alpha-1 }\end{array}$} & GABRA1 \\
\hline MOL000449 & \multicolumn{2}{|c|}{ Prostaglandin $\mathrm{G} / \mathrm{H}$ synthase 1} & PTGS1 \\
\hline MOL000359 & \multicolumn{2}{|c|}{ Nuclear receptor coactivator 2} & $\mathrm{NCOA}-2$ \\
\hline MOL000449 & \multirow{2}{*}{\multicolumn{2}{|c|}{$\begin{array}{l}\text { Beta-2 adrenergic receptor } \\
\text { Sodium channel protein type } 5 \\
\text { subunit alpha }\end{array}$}} & ADRB2 \\
\hline MOL000449 & & & SCN5A \\
\hline MOL000449 & \multicolumn{2}{|c|}{ Alpha-1B adrenergic receptor } & ADRA1B \\
\hline \multicolumn{4}{|c|}{$\begin{array}{l}\text { TABLE 4: GO BIOLOGICAL PROCESS ENRICHMENT } \\
\text { ANALYSIS OF TARGET }\end{array}$} \\
\hline $\begin{array}{l}\text { Biological } \\
\text { Process }\end{array}$ & Related Targets & $\mathbf{P}$ & Benjamini \\
\hline Neural regulation & $\begin{array}{ll}n & \text { PTGS2, ADRB2 }\end{array}$ & 0.00575 & 0.21 \\
\hline Cell cycle & $\begin{array}{l}\text { PGR, SCN5A, } \\
\text { ADRA1B }\end{array}$ & 0.01532 & 0.43 \\
\hline Apoptosis & $\begin{array}{l}\text { PTGS1, GABRA1, } \\
\text { SCN5A }\end{array}$ & 0.00784 & 0.20 \\
\hline $\begin{array}{l}\text { Inflammation } \\
\text { regulation }\end{array}$ & $\begin{array}{l}\text { PTGS2, ADRB2, } \\
\quad \text { NCOA-2 }\end{array}$ & 0.01074 & 0.38 \\
\hline $\begin{array}{l}\text { Cell } \\
\text { communication }\end{array}$ & $\begin{array}{l}\text { PTGS1, GABRA, } \\
\text { SCN5A }\end{array}$ & 0.00058 & 0.04 \\
\hline
\end{tabular}

enrichment analysis of KEGG signal pathway of YiyiFuzi-Baijiang powder 's anticolorectal cancer target (Table 5) showed that 8 therapeutic targets participate in 4 categories and 6 signal pathways, including neural regulation, apoptosis, cell cycle and inflammation regulation, as shown in Table 5, mainly including neuroactive ligand-receptor interaction, endocrine regulated calcium reabsorption, TGF- $\beta$ signaling pathway, Hedgehog signaling pathway, inflammatory 
TABLE 5: ENRICHMENT ANALYSIS OF TARGET KEGG METABOLIC PATHWAY

\begin{tabular}{lccc}
\hline Metabolic pathway & Related targets & P & Benjamini \\
\hline Neuroactive ligand-receptor interaction & PGR, NCOA-2, SCN5A & 0.032 & 0.45 \\
Endocrine regulated calcium reabsorption & NCoA-2, ADRA1B, SCN5A & 0.045 & 0.65 \\
TGF-B signaling pathway & PTGS2, ADRB2 & 0.061 & 0.72 \\
Hedgehog signaling pathway & SCN5A, ADRA1B & 0.015 & 0.33 \\
Inflammatory mediator regulation TRP channels & PTGS1, GABRA1, SCN5A & 0.050 & 0.24 \\
Cholinergic synapse & PTGS2, ADRB2 & 0.012 & 0.46 \\
\hline
\end{tabular}

mediator regulation TRP channels, cholinergic synapse. The results showed that the main active components of Yiyi-Fuzi-Baijiang powder were distributed in different metabolic pathways, and the possible mechanism of action was to coordinate and regulate each other.

Yiyi-Fuzi-Baijiang powder comes from one of the 4 classics of traditional Chinese medicine, synopsis of the Golden Chamber ulceration, carbuncle, intestinal abscess, soakage, pulse syndrome and treatment of the 18th: the intestine carbuncle is a disease where the body is wrong, the abdomen is urgent, according to it, if it is swollen, the abdomen is not accumulated, the body is not hot, the pulse number, this is the intestine has carbuncle pus, Yiyi-Fuzi-Baijiang powder main. This prescription is composed of Coix seed, aconite and Patrinia villosa. It was first recorded in the treatment of intestinal carbuncle. It is mainly used for the treatment of intestinal carbuncle and pus ${ }^{[17]}$. It has the function of eliminating carbuncle and removing pus, and strengthening yang qi. In modern times, it is used in intestinal, skin, gynecology, andrology and other diseases, as well as dry skin, abnormal keratosis and other diseases ${ }^{[18-20]}$. It is most commonly used in the treatment of abscess and purulent inflammation ${ }^{[18]}$. In the prescription, Coix seed is used to eliminate carbuncle and swelling, Patrinia villosa is used to clear heat, detoxify and discharge pus, while Fuzi Wenyang powder is used to relieve cold and pain. Modern pharmacology research showed that many monomers in Yiyi-Fuzi-Baijiang powder have anticancer effect.

In this investigation it was found that sitosterol $\alpha-1$, mandenol, (2R)-2,3-dihydroxypropyl (Z)octadec-9-enoate, sitosterol, stigmasterol, CLR, 11,14-eicosadienoic acid, deltoin, karakoline, karanjin, neokadsuranic acid $\mathrm{B}$, sitosterol, vilmorrianine $\mathrm{C}$, asperglaucide, acacetin, sinoacutine, $\beta$-sitosterol, sitosterol, stigmasterol as 19 main active ingredients in Coix, aconite and Patrinia, acting on progesterone receptor, prostaglandin $\mathrm{G} / \mathrm{H}$ synthase $2, \gamma$-aminobutyric acid receptor subunit $\alpha-1$, prostaglandin $\mathrm{G} / \mathrm{H}$ synthase 1 , nuclear receptor coactivator $2, \beta-2$ adrenergic receptor, sodium channel protein type 5 subunit $\alpha$, $\alpha-1 B$ adrenergic receptor several potential targets. These targets may play an important role in the antitumor network. The enrichment analysis of go biological process showed that the target genes of YiyiFuzi-Baijiang powder were involved in the biological processes of neural regulation, cell cycle, apoptosis, inflammation regulation, cell communication and so on. These biological processes are closely related to the development of nervous system and cancer cells and may be involved in the pathophysiological process of colorectal cancer. Some targets are related to cell cycle, apoptosis and inflammation control, which proved that the alkaloid extract of Yiyi-Fuzi-Baijiang powder has good analgesic and antiinflammatory effect, could regulate the central nervous system and has a certain central nervous inhibitory effect ${ }^{[21-23]}$. Therefore, YiyiFuzi-Baijiang powder can produce antitumor activity by inhibiting tumor growth and migration, regulating energy, regulating neural system multi phenotype intervention network mode, and has a certain relieving effect on cancer pain.

The results of KEGG metabolism pathway analysis showed that the related targets of the active components in the powder were, progesterone receptor, prostaglandin $\mathrm{G} / \mathrm{H}$ synthase $2, \gamma$-aminobutyric acid receptor subunit $\alpha-1$, prostaglandin $\mathrm{G} / \mathrm{H}$ synthase 1 , nuclear receptor coactivator $2, \beta-2$ adrenergic receptor, sodium channel protein type 5 subunit alpha, $\alpha-1 \mathrm{~B}$ adrenergic receptor participating in neuroactive ligandreceptor interaction, endocrine regulated calcium reabsorption, TGF- $\beta$ signalling pathway, Hedgehog signaling pathway, inflammatory mediator regulation TRP channels, cholinergic synapse signal pathway. Neuroactive ligand-receptor interaction ${ }^{[24]}$, endocrine regulated calcium reabsorption ${ }^{[25]}$, cholinergic synapse $^{[26]}$, Hedgehog signaling pathway ${ }^{[27]}$ are mainly related to the inflammatory reaction and the occurrence and development of cancer cells and participates in the process of cell cycle change and apoptosis. TGF- $\beta$ signaling pathway ${ }^{[28]}$ can affect PTGS2 -and ADRB2 gene to mediate brain activity, thus affecting the transmission of neural information. Inflammatory mediator regulation TRP channels ${ }^{[29]}$ mainly involved 
in cell communication and related to the growth and development of cancer cells. In this study, the target of Coix Fuzi-Baijiang powder in the treatment of colorectal cancer was involved as neuroactive ligandreceptor interaction (3 targets), endocrine regulated calcium reabsorption (3 targets), TGF- $\beta$ signalling pathway ( 2 targets), Hedgehog signalling pathway (2 targets), inflammatory mediator regulation TRP channels ( 3 targets) and cholinergic synapse ( 2 targets). It is suggested that the active components of Yiyi-FuziBaijiang powder may play an antiinflammatory and analgesic role by participating in the above pathway, thereby reducing the inflammatory response and regulating the nervous system.

In conclusion, this study described the reticular relationship among the main active components, targets and pathways of Yiyi-Fuzi-Baijiang powder. It was found that Yiyi-Fuzi-Baijiang powder not only has neuromodulatory effect, but also has multi-dimensional pharmacological effects such as anticancer and antiinflammatory. However, network pharmacology research is inseparable from the integrity and practicability of related databases and there are some differences between the technology of molecular simulation docking and the environment in vivo. Therefore, the relevant targets and molecular mechanisms of the research still need further experimental verification.

\section{Acknowledgements:}

The authors thank the training program for core talents in TCM innovation of China and the 4th China National TCM Excellent Talents Training Projects 2017 supporting our study.

\section{Funding:}

This work was supported by the Natural Science Foundation of Jiangsu Province (BK20171096).

\section{Conflict of interest:}

All authors report no conflicts of interest in this work.

\section{REFERENCES}

1. Ye W, Ling S, Liu RY, Pan ZZ, Wang G, Gao S, et al. Exome sequencing reveals the genetic landscape and frequent inactivation of PCDHB3 in Chinese rectal cancers. J Pathol 2018;24:222-34.

2. Zhao LZ, Ma DW, Zhang XP. Current status of colorectal cancer screening at home and abroad and progress of noninvasive screening methods. Tianjin Med J 2019.

3. Chubak J, Boudreau DM, Rulyak SJ, Mandelson MT. Colorectal cancer risk in relation to antidepressant medication use. Int J Cancer 2011;128:227-32.

4. Tilg H, Adolph TE, Gerner RR, Moschen AR. The intestinal microbiota in colorectal cancer. Cancer cell 2018 ;33:954-64.

5. Bychkov D, Linder N, Turkki R, Nordling S, Kovanen PE, Verrill $\mathrm{C}$, et al. Deep learning based tissue analysis predicts outcome in colorectal cancer. Sci Rep 2018;8:3395.

6. Alsamak FF, Abdulamir AS, Mahdi LK, Alnaib K, Bakar FA. Association of Helicobacter pylori with colorectal cancer development. Asian Biomed 2010;4:609-18.

7. Draht M, Goudkade D, Koch A, Grabsch HI, Weijenberg MP, van Engeland M, et al. Prognostic DNA methylation markers for sporadic colorectal cancer: a systematic review. Clin Epigenetics 2018;10:35.

8. Schwingshackl L, Schwedhelm C, Hoffmann G, Knüppel $\mathrm{S}$, Laure Preterre A, Iqbal K, et al. Food groups and risk of colorectal cancer. Int J Cancer 2018;142:1748-58.

9. Bang-Cai W. Cases of treating intractable and severe disease by classic prescription. J Tradit Chin Med Pharm 2010;25:54042.

10. Muhammad J, Khan A, Ali A, Fang L, Yanjing W, Xu Q, et al. Network Pharmacology: Exploring the Resources and Methodologies. Curr Top Med Chem 2018;18:949-64.

11. Ru J, Li P, Wang J, Zhou W, Li B, Huang C, et al. TCMSP: a database of systems pharmacology for drug discovery from herbal medicines. J Cheminformatics 2014;6:13.

12. UniProt: a hub for protein information. Nucleic Acids Res 2015;43:D204-D12.

13. Netherlands S. OMIM Encyclopedia of Genetics, Genomics, Proteomics and Informatics. Springer Netherlands 2008.

14. Gao Z, Li H, Zhang H, Liu X, Kang L, Luo X, et al. PDTD: a web-accessible protein database for drug target identification. BMC Bioinformatics 2008;9:104.

15. Hewett M, Oliver DE, Rubin DL, Easton KL, Stuart JM, Altman RB, et al. PharmGKB: the Pharmacogenetics Knowledge Base. Nucleic Acids Res 2002;30:163-65.

16. Kohl M, Wiese S, Warscheid B. Cytoscape: Software for Visualization and Analysis of Biological Networks. Methods Mol Biol 2011;696:291-303.

17. Li DM, Yan CH. Two principles for the treatment of coiyi Fuzi and Baijiang powder . J Tradit Chin Med 2006;25:494-95.

18. Chey WY, Kunze GY, Shah AN. Observations on therapeutic effect of Infliximab on ulcerative colitis. Am J Gastroenterol 2001;96:S288.

19. Fang J, Chen J, Peng JW. Based on Nrf2 pathway, to explore the mechanism of Coix Fuzi Patrinia powder in the treatment of ulcerative colitis. J Exp Pharmacol 2018.

20. Xiao YY, Wang G. analysis of syndrome differentiation, prescription and recurrence of 121 cases of verruca plana cured clinically. 2018 national academic annual meeting of dermatovenereal diseases with integrated traditional and Western medicine.

21. Van Hee VC, Adar SD, Szpiro AA, Barr RG, Roux AD, Bluemke DA, et al. Common Genetic Variation, Residential Proximity to Traffic Exposure, and Left Ventricular Mass: The Multi-Ethnic Study of Atherosclerosis. Environ. Health Perspect 2010;118:962-9.

22. Novak P, Cente M, Kosikova N, Augustin T, Kvetnansky $\mathrm{R}$, Novak M, et al. Stress-Induced Alterations of Immune Profile in Animals Suffering by Tau Protein-Driven Neurodegeneration. Cell Mol Neurobiol 2018;38:243-59.

23. Valencia DM, Naranjo CA, Parra MV, Caro MA, Valencia $\mathrm{AV}$, Jaramillo CJ, et al. Association and interaction of AGT, AGTR1, ACE, ADRB2, DRD1, ADD1, ADD2, ATP2B1, 
TBXA2R and PTGS2 genes on the risk of hypertension in Antioquian population. Biomedica 2013;33:598-14.

24. Day CJ, Hartley-Tassell LE, Korolik V. In: Identification of Ligand-Receptor Interactions: Ligand Molecular Arrays, SPR and NMR Methodologies. Campylobacter jejuni. New York: Springer 2017;51-63.

25. Wu S, Gu Y, Huang Y, Wong TC, Ding H, Liu T, et al. Novel Biomarkers for Non-functioning Invasive Pituitary Adenomas were Identified by Using Analysis of microRNAs Expression Profile. Biochem Genet 2017;55:253-67.

26. Legay C. Congenital myasthenic syndromes with acetylcholinesterase deficiency, the pathophysiological mechanisms. Ann NY Acad Sci 2018;1413:104-10.

27. Varjosalo M, Taipale J. Hedgehog signaling. J Cell Sci 2007;120:3-6.
28. Javle $\mathrm{M}$, Li Y, Tan D, Dong X, Chang $\mathrm{P}$, Kar S, et al. Biomarkers of TGF- $\beta$ signaling pathway and prognosis of pancreatic cancer. PloS One. 2014;9:e85942.

29. Barnes PJ, Chung KF, Page CP. Inflammatory mediators and asthma. Pharmacol Reviews 1988;40:49-84.

This is an open access article distributed under the terms of the Creative Commons Attribution-NonCommercial-ShareAlike 3.0 License, which allows others to remix, tweak, and build upon the work non-commercially, as long as the author is credited and the new creations are licensed under the identical terms

This article was originally published in a special issue, "Biomedical applications in Pharmaceutical Sciences" Indian J Pharm Sci 2020:82(2)Spl issue3;135-141 